\title{
Efficacy of a Topical Agent Containing Tranexamic Acid, Niacinamide and Kojic Acid in Melasma
}

\author{
Xiaoxue Xing, Li Chen, Zhongyi Xu, Shanglin Jin, Chengfeng Zhang*, and Leihong Xiang* \\ *Department of Dermatology, Huashan Hospital, Fudan University, Shanghai 200040, PR China
}

*Corresponding author: Zhang C, Xiang L, Department of Dermatology, Huashan Hospital, Fudan University, Shanghai 200040, PR China, Tel: +86 21 52889999; Tax: +86 21 52887783; E-mail: flora_xiang@vip.163.com, e3dangdang@hotmail.com

Received: 04 Jan, 2021 | Accepted: 11 Jan, 2021 | Published: 19 Jan, 2021

Citation: Xing X, Chen L, Xu Z, Jin S, Zhang C, et al. (2021) Efficacy of a Topical Agent Containing Tranexamic Acid, Niacinamide and Kojic Acid in Melasma. J Clin Cosmet Dermatol 5(1): dx.doi.org/10.16966/2576-2826.160

Copyright: (c) 2021 Xing X, et al. This is an open-access article distributed under the terms of the Creative Commons Attribution License, which permits unrestricted use, distribution, and reproduction in any medium, provided the original author and source are credited.

\section{Abstract}

Background: Melasma is a common pigmentary disorder in Asian women.

Objective: To evaluate the efficacy of a new topical agent containing $3 \%$ tranexamic acid, $5 \%$ niacinamide, and $1 \%$ kojic acid in Chinese melasma patients.

Methods: Twenty-seven Chinese patients ( 26 women and $1 \mathrm{man}$ ) with facial melasma were enrolled. Participants applied the agent to the entire face twice a day for 12 weeks, and treatment outcomes were evaluated using a 5-grade clinical assessment scale. At weeks 2, 4, 8, and 12 of therapy, standard photographs of the patients were acquired with a skin imaging system and the melanin index (MI) of the lesions and normal skin were measured.

Results: Twenty-two patients completed the therapy. Among them, $86.36 \%$ cases had improved pigmentation according to the clinical assessment scale after the treatment. Compared with baseline, the MI of the lesions and the ratio of MI to normal skin declined significantly by week 12 , with no obvious decrease in normal skin's MI.

Conclusion: The novel agent containing $3 \%$ tranexamic acid, $5 \%$ niacinamide, and $1 \%$ kojic acid is safe and effective in the treatment of Chinese melasma patients, with greater effects on the hyper-pigmented compared with normal skin.

Keywords: Melasma; Tranexamic acid; Niacinamide; Kojic acid; Topical therapy

\section{Introduction}

Melasma, a commonly acquired melanogenic disease with a high incidence in Asian women, is characterized by brown to grey-brown patches on the temporal region of the face, cheeks, and forehead [1]. Although several causes have been elucidated for melasma, including UV exposure, genetic predisposition, mental stress, endocrine factors, and skin inflammation [2], more evidence is needed to fully understand its pathogenesis. Sunscreens, oral multivitamins, Tranexamic Acid (TA), and other topical agents or procedures such as hydroquinone, laser therapy, and chemical peels are efficacious in the treatment of melasma [3]. Despite the multiformity of treatments, a large number of cases remain recurrent or refractory [4], making melasma treatment challenging for dermatologists.

Oral TA has been confirmed as an effective treatment option for melasma, especially in recurrent and refractory cases [5]. However, due to concerns regarding the possible systematic side effects of oral therapy [6], many Chinese patients prefer topical treatment. Several topical therapies have been shown to affect melasma and melanocyte activities [7]. For example, a TA solution combined with the use of a thulium $1927 \mathrm{~nm}$ fractional laser for micro needling or a topical liposomal TA agent have been developed to improve pigmentation to varying degrees [8]. Nicotinamide, also known as niacinamide, is another well-known depigmentary agent [9], which suppresses melanosome transfer from melanocytes to keratinocytes [10]. In addition, kojic acid (5-hydroxy-2-(hydroxymethyl)-4-pyrone) exerts skin-lightening effects through its induction of IL-6 production in keratinocytes and the subsequent inhibition of melanocyte activities [11].

With the expectation of cumulative effects of the three agents, this study aimed to evaluate the efficacy and safety of a novel topical serum containing 3\% tranexamic acid, $5 \%$ niacinamide, and $1 \%$ kojic acid in the treatment of Chinese melasma patients.

\section{Methods}

\section{Patients}

This study enrolled 27 Chinese patients with bilateral facial melasma (26 females and 1 male) at the dermatology outpatient clinic of Huashan Hospital from March 2019 to April 2019. Bilateral facial melasma patients aged 18 to 65 years with Fitzpatrick skin type III-IV were included. Exclusion criteria were treatment history 
of oral tranexamic acid, laser therapy, and/or other topical agents for melasma within 1 month before enrollment. All patients were evaluated by dermatologists for recruitment eligibility and informed of the advantages and risks of the therapy before signing the written consent. This clinical study was approved by the Medical Ethics and Human Research Committee of Huashan Hospital (No. 2017-438) and conducted in accordance with the ethical guidelines of the Declaration of Helsinki. In total, 22 out of 27 patients completed the therapy and 5 visits, while 5 dropped out or missed some of the visits due to distance.

\section{Study design}

Melasma all patients were shown how to apply the solution containing 3\% tranexamic acid, 5\% niacinamide, 1\% kojic acid, and $5 \%$ hydroxyethylpiperazine ethane sulfonic acid (SkinCeuticals, USA, lot number: J20181555), covering the entire face twice a day. The duration of therapy was 12 weeks. Patients were also asked to apply a sunscreen cream (SPF 50, Skin Ceuticals, USA, lot number: J20150626) throughout the study.

\section{Clinical assessment}

Patients were instructed to visit the outpatient clinic at baseline $0,2,4,8$, and 12 weeks during the treatment for examinations. Two dermatologists assessed Modified Melasma Area and Severity Index (mMASI) scores independently at each visit. The final clinical outcome was evaluated by dermatologists at the end of the 12-week treatment using a 5-grade clinical assessment scale: "almost clear", > 75\% improvement; "marked improvement", 51-75\% improvement; "moderate improvement", 25-50\% improvement; "minimal improvement", 0-25\% improvement; "stable or worsening", no improvement or aggravation. The Melanin Index (MI) of lesion areas and normal skin were evaluated at $0,2,4,8$, and 12 weeks using a derma spectrophotometer (Mexameter, Cortex Technology, Denmark). Standard clinical photographs were taken by a skin imaging system (VISIA Complexion Analysis System, Canfield Scientific, Parsippany, USA ) at $0,2,4,8$, and 12 weeks.

\section{Statistical analysis}

SPSS v23.0 (IBM, Chicago, USA) was used for statistical analysis. Paired sample t-test was applied to compare mMASI scores, and differences in MI in lesion and non-lesion areas at baseline and at 2, 4, 8 , and 12 weeks, respectively.

\section{Results}

\section{Clinical assessment}

Among the 22 patients who completed the therapy and followup visits, $36.4 \%$ ( 8 out of 22 ) showed marked improvement, $22.7 \%$ (5 out of 22) achieved moderate improvement and 27.3\% (6 out of 22) had mild improvement, while $13.6 \%$ (3 out of 22) showed no improvement or worsening symptoms (Figure 1). The overall effective rate of $86.36 \%$ was defined by the number of patients who achieved marked, moderate, or mild improvement divided by the total number of patients. Representative clinical pictures of two patients are shown in figure 2, illustrating marked improvement of lesions.

\section{Modified melasma area and severity index}

As shown in figure 3, mMASI scores evaluated by two independent dermatologists decreased gradually throughout the study. Two weeks after treatment initiation, mMASI scores were significantly decreased compared with baseline values $(\mathrm{P}=0.044$, which was very close to the cut-off value for significance of 0.05 ). At 4,8 , and 12 weeks, very significant reductions in mMASI scores compared with baseline values were observed, indicating a gradual improvement of melasma throughout the treatment ( 4 weeks, $\mathrm{P}<0.01 ; 8$ weeks, $\mathrm{P}<0.001 ; 12$ weeks, $\mathrm{P}<0.001)$.

\section{Melanin index changes}

After two weeks of treatment, the MI in the lesion area tended to decrease compared with baseline, but not significantly (Figure 4a). At 4,8 , and 12 weeks after enrollment, MI values in the lesion areas were reduced significantly compared with baseline ( 4 weeks, $\mathrm{P}<0.001 ; 8$ weeks, $\mathrm{P}<0.001 ; 12$ weeks, $\mathrm{P}<0.01$ ) (Figure $4 \mathrm{a}$ ).

For assessing the overall whitening effect of the treatment, the MI of the non-lesion area was determined for each patient at every visit. As illustrated in figure $4 \mathrm{~b}$, the MI of normal skin decreased significantly at 2 and 4 weeks after treatment initiation ( 2 weeks, $\mathrm{P}<0.01 ; 4$ weeks, $\mathrm{P}<0.01)$, but slightly increased at 8 and 12 weeks $(\mathrm{P}>0.05)$.

The ratio of mean MI of lesion area by that of normal skin was assessed to determine whether the agent functioned mostly through the overall whitening effect or mostly affected the pigmented area. The results demonstrated significant differences after 4, 8, and 12 weeks of therapy ( 4 weeks, $\mathrm{P}<0.01 ; 8$ weeks, $\mathrm{P}<0.001 ; 12$ weeks, $\mathrm{P}<0.001$ ) (Figure $4 \mathrm{c}$ ), suggesting that the agent was more effective in lightening the pigmented skin areas.

\section{Adverse effects}

One patient dropped out of the study 2 days after treatment initiation due to facial erythema and itching; however, the symptoms subsided after stopping topical application. No other severe adverse effects were observed during the trial.

\section{Discussion}

This study has shown the beneficial effects of a novel topical agent containing $3 \%$ TA, $5 \%$ niacinamide, and $1 \%$ kojic acid in the treatment of melasma patients, with significant improvement of pigmentation by the end of the 12-week treatment. TA, as an inhibitor of plasmin, could inhibit the activation of plasminogen and mitigate UV radiationinduced pigmentation in the physiological exchange between epidermal keratinocytes and melanocytes [12]. Niacinamide, another classic skin-lightening agent, could decrease melanosome transfer and
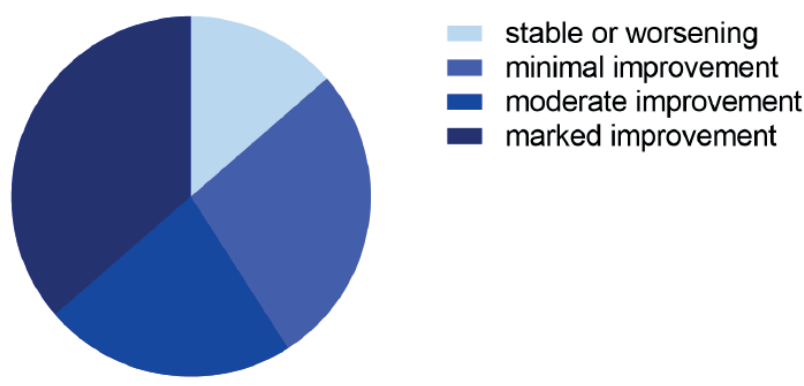

Total $=\mathbf{2 2}$

Figure 1: Clinical outcomes of all patients at the endpoint evaluated by a 5 -grade clinical assessment scale.

"Almost clear", >75\% improvement, "marked improvement", 5175\% improvement, "moderate improvement", 25-50\% improvement, "minimal improvement", 0-25\% improvement, "stable or worsening", no improvement or aggravation. 


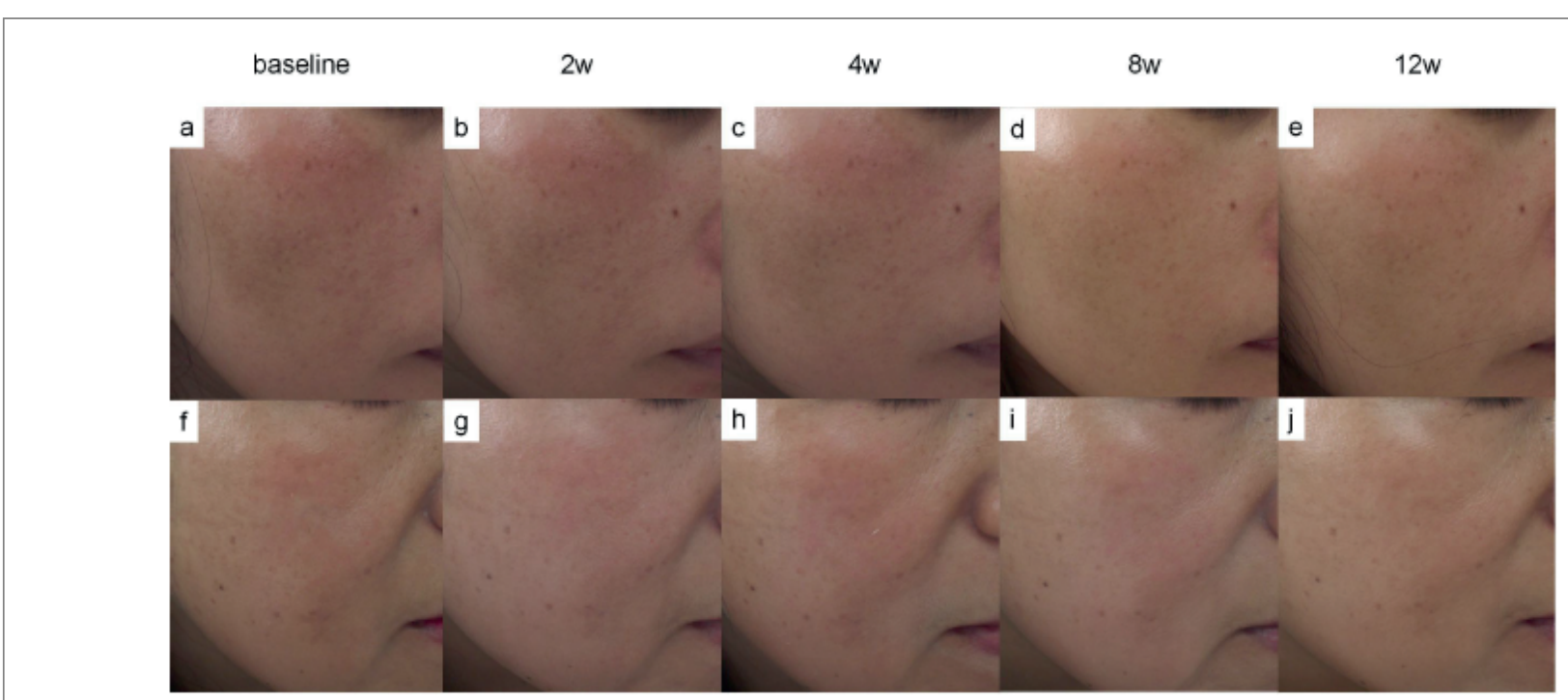

Figure 2: Representative photographs of two patients. a, b, c, d and e. Patient 1 at weeks $0,2,4,8$ and 12 of treatment, respectively. $f, g, h, i$, and j. Patient 2 at weeks $0,2,4,8$ and 12 of treatment, respectively.

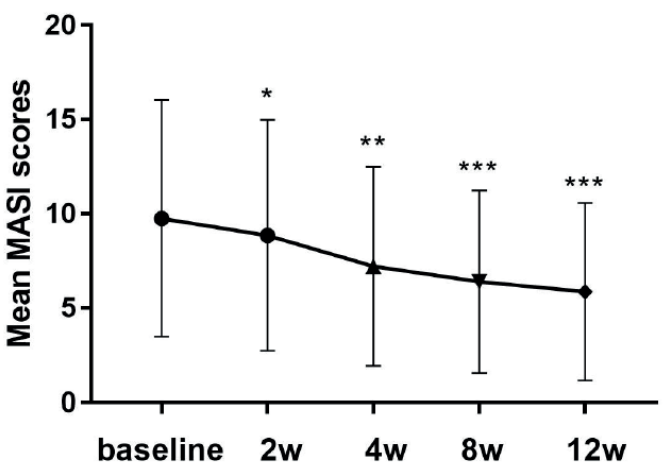

Figure 3: Changes of mMASI scores evaluated by two independent dermatologists over time. Week $2, \mathrm{P}=0.044$, week $4, \mathrm{P}<0.01$, weeks 8 and $12, \mathrm{P}<0.001$.

have anti-inflammatory functions [13]. Kojic acid is known to have inhibitory effects on the activity of tyrosinase and melanogenesis [14]. Whereas the above three ingredients of the formulation applied in the current study have previously shown their whitening effects respectively, our results demonstrated a synergistic effect of these three depigmentary drugs, and more importantly, with good skin tolerance, and low incidence of severe side effects.

In accordance with our results, the similar formulation was found to be effective and well-tolerated in the treatment of facial dyschromia such as melasma and post-inflammatory hyperpigmentation in a female population in Brazil [15]. However, in the current study, we evaluated the efficacy and safety of the serum in a single ethnic population. The conclusions of our trial are therefore more important for clinical practice in Asian populations, especially regarding tolerance. Moreover, we evaluated the MI index in, affected area and normal skin as well as the ratio between them and found that the formula could significantly decrease the MI index in the affected area, with no obvious change in $\mathrm{MI}$ in the skin without lesions at the final follow-up. Together, with a gradient descent of ratio between MI in the affected and non-affected areas, it seemed that the agent had potent effects on areas of hyper pigmentation rather than effecting overall whitening. However, given that the trial was conducted during the summer when there is severe UV exposure, we recognize that the evaluation of overall whitening could be impacted. Further study during the winter months would be required for ruling out the effects caused by UV exposure. Furthermore, we observed a statistically significant decrease in mMASI after 2 weeks compared to baseline, but the statistical significance was very close to 0.05 . Combined with other results that showed a significant improvement after 4-weeks of treatment according to MI and clinical photographs, we would prefer to conclude that a clinically significant improvement occurred after 4 weeks of application in the Chinese population. This differs from the significant change after 2-week treatment in the Brazilian study.

Although treatment of melasma remains a big challenge, therapy such as oral TA treatment has proved to be effective in most cases [16]. However, oral TA has yet served as off-lable use in treating melasma in China. Other topical therapies, such as $2 \%$ hydroquinone, may be effective but can cause irritation and worsen pigmentation in some cases [17]. For patients with low compliance with oral therapy and sensitive or telangiectatic skin, the current formulation under study with $3 \%$ TA, $5 \%$ niacinamide, and $1 \%$ kojic acid could be a choice given its efficacy and tolerance.

Topical TA is effective in the treatment of melasma and rosacea by regulating the immune response and angiogenesis $[18,19]$. As increased vascularization plays a role in the pathogenesis of melasma [20], we expected the topical agent might exert promising effects not only on hyperpigmentation but also on erythema, especially in patients with vascular ectasia. In this study, we observed a depigmenting effect in patients with pigmentary and vascular types, with alleviation of erythema in some cases (Figure 2). Future clinical studies designed with sub-group analysis and evaluation of erythema index are needed to further reveal the function of this novel agent on erythema and its pathology. In addition, a longer follow-up after the endpoint to evaluate the recurrence rate is needed.

In conclusion, a new formula consisting of 3\% TXA, 1\% kojic acid, and $5 \%$ niacinamide is effective and safe for the treatment of melasma 
a

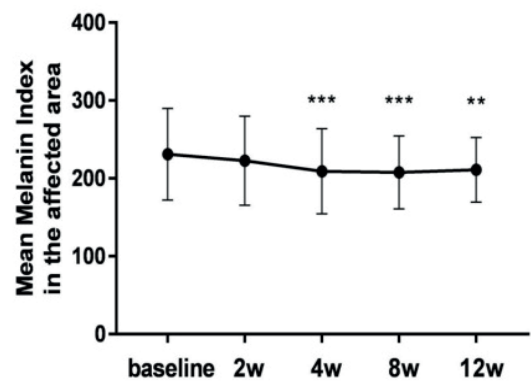

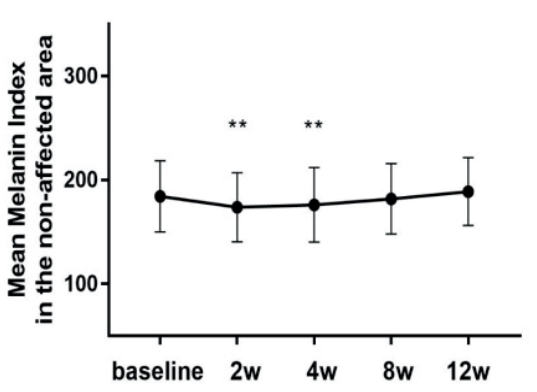

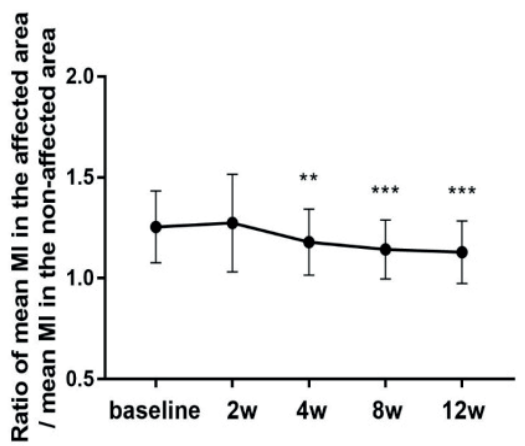

Figure 4: Changes of Melanin Index (MI) in the affected and non-affected areas, and their ratios over time.

(a) Changes of $\mathrm{Ml}$ in the affected area throughout the study (b) Changes of $\mathrm{MI}$ in the non-affected area throughout the study (c) Changes of the ratio of $\mathrm{MI}$ in the affected area divided by that of the non-affected area throughout the study. ${ }^{* * P} \mathrm{P}<0.01$. ${ }^{* * *} \mathrm{P}<0.001$.

in a Chinese population, with an observed improvement generally beginning at week 4 and a significant change by week 12 .

\section{Funding}

This work was supported by grants from Natural Science Foundation of China (No.81903243, and 81602775) and Shanghai Sailing Program (No.19YF1404800).

\section{Disclosure Statement}

These three authors contributed equally to this work. The authors have no conflict of interest to declare.

\section{Acknowledgments}

This work was supported by grants from the Natural Science Foundation of China (No. 81903243, and 81602775) and Shanghai Sailing Program (No. 19YF1404800).

\section{Declaration of Interest}

The authors report no conflicts of interest.

\section{References}

1. Lee BW, Schwartz RA, Janniger CK (2017) Melasma. G Ital Dermatol Venereol 152: 36-45.

2. Lee AY (2015) Recent progress in melasma pathogenesis. Pigment Cell Melanoma Res 28: 648-660.

3. Suggs AK, Hamill SS, Friedman PM (2018) Melasma: update on management. Semin Cutan Med Surg 37: 217-225.

4. Kwon SH, Na JI, Choi JY, Park KC (2019) Melasma: updates and perspectives. Exp Dermatol 28: 704-708.

5. Lee HC, Thng TGS, Goh CL (2016) Oral tranexamic acid (TA) in the treatment of melasma: a retrospective analysis. J Am Acad Dermatol 75: 385-392.

6. Sheu SL (2018) Treatment of melasma using tranexamic acid: what's known and what's next. Cutis 101: E7-E8.

7. Ogbechie-Godec OA, Elbuluk N (2017) Melasma: an up-to-date comprehensive review. Dermatol Ther (Heidelb) 7: 305-318.

8. Taraz M, Niknam S, Ehsani AH (2017) Tranexamic acid in treatment of melasma: A comprehensive review of clinical studies. Dermatol Ther 30: e12465.
9. Rolfe HM (2014) A review of nicotinamide: treatment of skin diseases and potential side effects. J Cosmet Dermatol 13: 324-328.

10. Hakozaki T, Minwalla L, Zhuang J, Chhoa M, Matsubara A, et al. (2002) The effect of niacinamide on reducing cutaneous pigmentation and suppression of melanosome transfer. Br J Dermatol 147: 20-31.

11. Choi H, Kim K, Han J, Choi H, Jin SH, et al. (2012) Kojic acid-induced IL-6 production in human keratinocytes plays a role in its antimelanogenic activity in skin. J Dermatol Sci 66: 207-215.

12. Perper M, Eber AE, Fayne R, Verne SH, Magno RJ, et al. (2017) Tranexamic acid in the treatment of melasma: a review of the literature. Am J Clin Dermatol 18: 373-381.

13. Navarrete-Solís J, Castanedo-Cázares JP, Torres-Álvarez B, OrosOvalle C, Fuentes-Ahumada C, et al. (2011) A double-blind, randomized clinical trial of niacinamide $4 \%$ versus hydroquinone $4 \%$ in the treatment of melasma. Dermatol Res Pract 2011: 379173.

14. Berardesca E, Rigoni C, Cantù A, Cameli N, Tedeschi A, et al. (2020) Effectiveness of a new cosmetic treatment for melasma. J Cosmet Dermatol 19: 1684-1690.

15. Desai S, Ayres E, Bak H, Manco M, Lynch S, et al. (2019) Effect of a tranexamic acid, kojic acid, and niacinamide containing serum on facial dyschromia: a clinical evaluation. J Drugs Dermatol 18: 454459.

16. Del Rosario E, Florez-Pollack S, Zapata L Jr, Hernandez K, Tovar-Garza A, et al. (2018) Randomized, placebo-controlled, double-blind study of oral tranexamic acid in the treatment of moderate-to-severe melasma. J Am Acad Dermatol 78: 363-369.

17. Cheong KAh, Kim HJ, Kim JY, Kim CH, Lim WS, et al. (2014) Retinoic acid and hydroquinone induce inverse expression patterns on cornified envelope-associated proteins: implication in skin irritation. J Dermatol Sci 76: 112-119.

18. Zhu JW, Ni YJ, Tong XY, Guo X, Wu XP, et al. (2020) Tranexamic acid inhibits angiogenesis and melanogenesis in vitro by targeting VEGF receptors. Int J Med Sci 17: 903-911.

19. Li Y, Xie H, Deng Z, Wang B, Tang Y, et al. (2019) Tranexamic acid ameliorates rosacea symptoms through regulating immune response and angiogenesis. Int Immunopharmacol 67: 326-334.

20. Kim EH, Kim YC, Lee ES, Kang HY (2007) The vascular characteristics of melasma. J Dermatol Sci 46: 111-116. 